\title{
Left atrial myxoma with aortic regurgitation
}

\author{
P. D. V. BOURDILLON 1 , J. L. MONRO, AND A. M. JOHNSON \\ From the Wessex Cardiac and Thoracic Centre, Western Hospital, Southampton
}

SUMMARY A 59-year-old woman with a left atrial myxoma was shown to have moderate aortic regurgitation. At operation for removal of the myxoma it was found to be attached to the atrial septum close to the aortic root. It is suggested that the aortic regurgitation was caused by distortion of the aortic root caused by traction upon it from the myxoma as it moved downward into the left ventricle in diastole. Observations are made on the echocardiographic findings which helped in the diagnosis of the left atrial myxoma.

Rose et al. (1974) described a case in which a left atrial myxoma was associated with aortic regurgitation. They suggested that the aortic valve was regurgitant because of direct trauma to it from the left atrial tumour prolapsing through the mitral valve. We report here a further case of a left atrial myxoma with aortic regurgitation which appeared to result from distortion of the aortic root caused by the downward movement in diastole of the myxoma which had a pedicle attached to the atrial septum close to the aortic root.

\section{Case report}

The patient, a 59-year-old white woman, presented with a history of intermittent palpitation and breathlessness on moderate exertion for about 5 years. The palpitation had been persistent for a year. There was no history of rheumatic fever. About 6 months before admission she was diagnosed as having mitral stenosis on the basis of the physical findings of a mitral facies, established atrial fibrillation, a soft delayed diastolic murmur, and an early opening snap. The electrocardiogram confirmed the presence of atrial fibrillation and the chest $x$-ray film showed an enlarged left atrium with some calcification in the region of the mitral valve. She was treated with digoxin, a thiazide diuretic, and oral anticoagulants. Because of persistent symptoms of palpitation and breathlessness she was referred to this hospital for further investigation.

Examination again revealed the physical findings

'Present address: National Heart Hospital, Westmoreland Street, London W1M 8BA. already described and in addition it was noted that the first heart sound and the added sound which was interpreted as an opening snap were soft. No immediate diastolic murmur was heard and the blood pressure was $140 / 95 \mathrm{mmHg}$.

Echocardiography was performed using a Smith Kline Ekoline 20 and a $2.25 \mathrm{MHz}$ transducer, focused at $5 \mathrm{~cm}$ and recorded on a Cambridge strip-chart recorder.

The transducer was placed at the left sternal border in the fourth intercostal space and was directed posterolaterally so as to produce a clear echo from the anterior leaflet of the mitral valve. An abnormal echo was obtained posterior to this and moving more or less in parallel with it (Fig. 1A, left, and Fig. 1B). On slowly changing the direction of the ultrasonic beam, sweeping superiorly and medially, mitral-aortic continuity was shown and the abnormal echo was seen to be continuous with an abnormal echo posterior to the aortic root, though this was less well defined (Fig. 1A, right). The mitral valve echogram, recorded nearer to the tip of the anterior leaflet of the mitral valve (Fig. 1B), shows a fine fluttering movement in diastole which is not seen in the abnormal echo posterior to it. The diastolic closure rate of the anterior leaflet of the mitral valve varied between $80 \mathrm{~mm} / \mathrm{s}$ and $100 \mathrm{~mm} / \mathrm{s}$.

Left and right heart catheterisation showed an end-diastolic gradient across the mitral valve of approximately $5 \mathrm{mmHg}$ though this appeared to be variable during the course of the procedure. Cine-left ventriculography showed good left ventricular contractility with trivial mitral regurgitation. Root aortography with the tip of the catheter well 
clear of the aortic valve indicated the presence of moderate (grade 2/3) aortic regurgitation. The aortic root was seen to be deformed in diastole (Fig. 2A and 2B). Pulmonary angiography with follow through to the left atrium showed a large left atrial mass which moved towards the mitral valve orifice in diastole and appeared to be pedunculated at its upper pole (Fig. 2C and 2D). Some calcification was noted superimposed on the left

atrium but moving separately from the valves, the coronary arteries, and the left atrial mass.

Immediate surgery was advised and carried out and therefore further echocardiographic studies could not be undertaken. A left atrial myxoma was found. It had a greenish gelatinous appearance with an impression on it where part of it had been pushed into the mitral valve, and measured about $3.5 \times 4.5 \mathrm{~cm}$ in cross-sectional diameters and
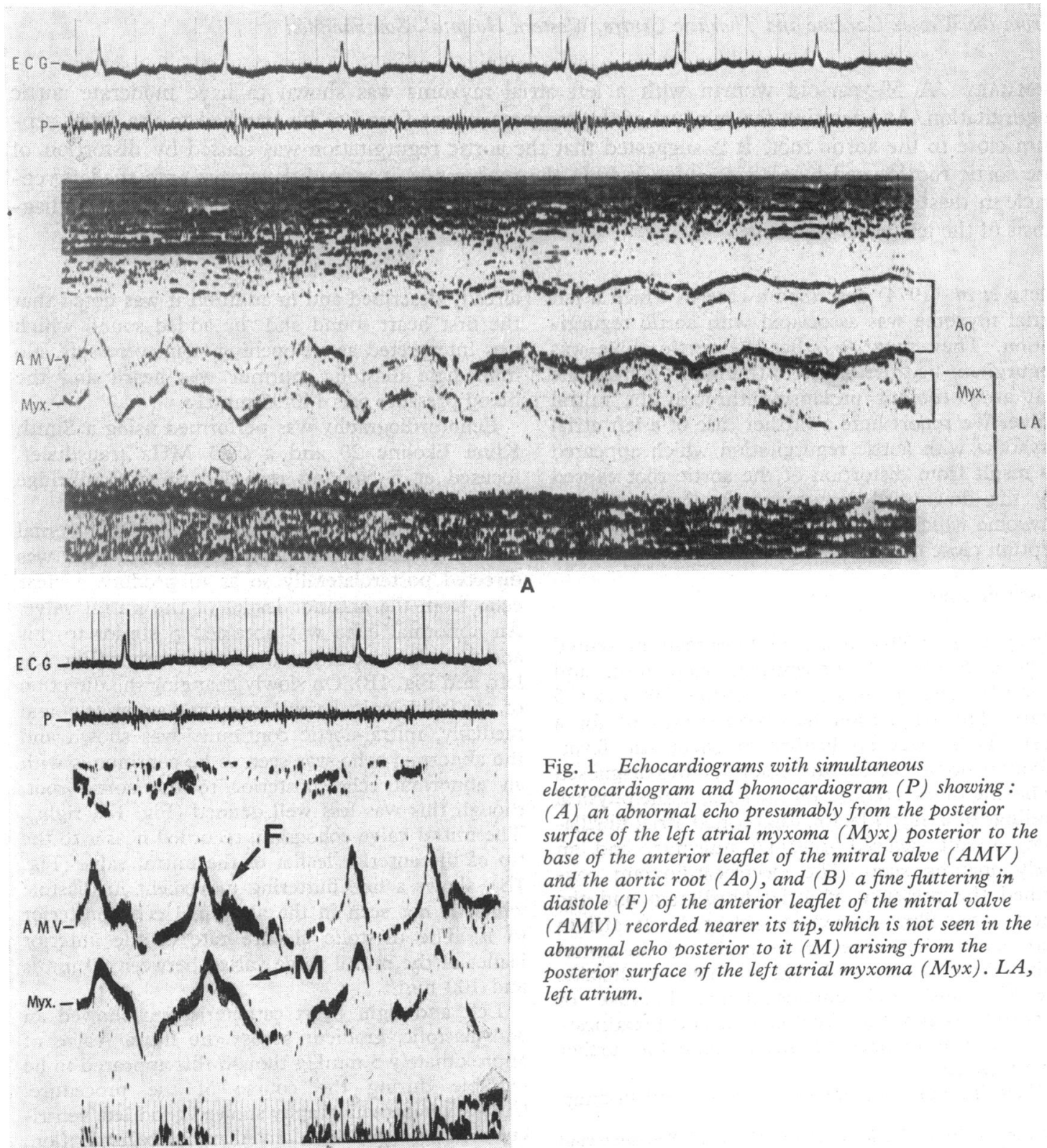

Fig. 1 Echocardiograms with simultaneous electrocardiogram and phonocardiogram $(P)$ showing: (A) an abnormal echo presumably from the posterior surface of the left atrial myxoma (Myx) posterior to the base of the anterior leaflet of the mitral valve $(A M V)$ and the aortic root $(A o)$, and $(B)$ a fine fluttering in diastole $(F)$ of the anterior leaflet of the mitral valve $(A M V)$ recorded nearer its tip, which is not seen in the abnormal echo Dosterior to it $(M)$ arising from the posterior surface of the left atrial myxoma $(M y x) . L A$, left atrium. 

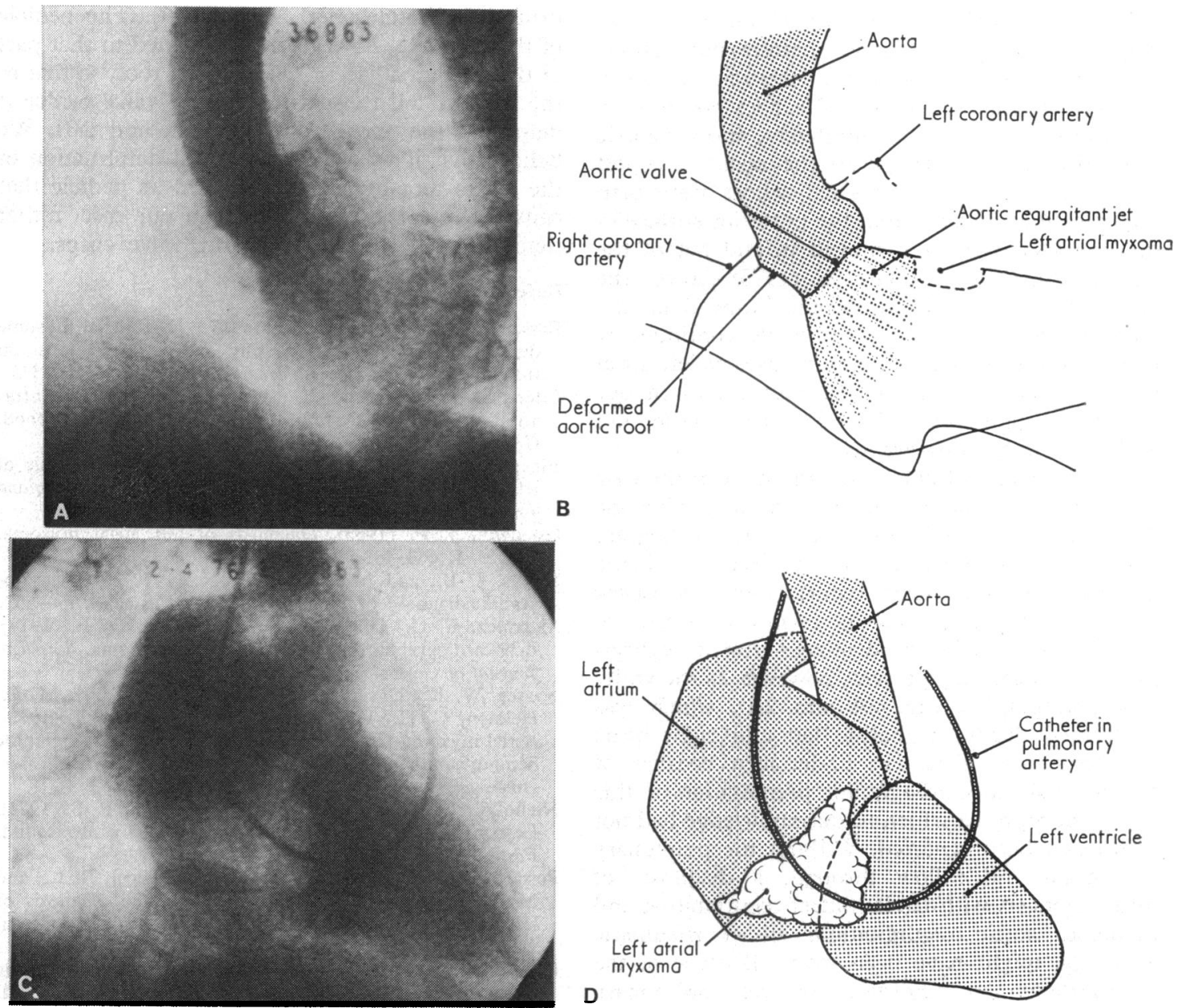

B

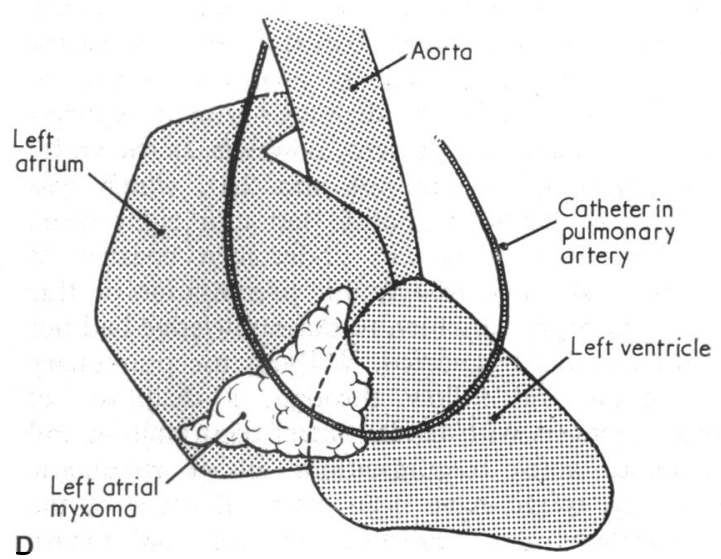

Fig. 2 Angiograms. (A) Root aortography in the left anterior oblique projection. (B) Illustration of the angiographic anatomy seen in $(A)$. (C) Left atrial phase of the pulmonary angiogram in the right anterior oblique projection. (D) Illustration of the angiographic anatomy seen in (C).

about $5 \mathrm{~cm}$ in length. It was attached by a pedicle approximately $1.7 \mathrm{~cm}$ in diameter to the left side of the atrial septum adjacent to the aortic root. The tumour did not extend into the right atrium. The part of the atrial septum bearing the pedicle was excised and this and the tumour were removed. A hole in the septum about $2.5 \mathrm{~cm}$ in diameter was repaired with a 'dacron' patch. The mitral valve was normal and after removal of the myxoma there was no aortic regurgitation seen on looking through the mitral valve.

After operation the patient remained in atrial fibrillation and DC cardioversion performed on the 11th postoperative day resulted in a junctional rhythm. This subsequently reverted to sinus rhythm and the patient has remained well with no residual signs of aortic or mitral valve disease. She declined to be reinvestigated.

\section{Discussion}

The diagnosis of left atrial myxoma is notoriously difficult and is often missed (Goodwin, 1963). As in our case, an initial diagnosis of mitral stenosis is frequently made. Echocardiography has been helpful in establishing the diagnosis (Effert and Domanig, 1959; Schattenberg, 1968; Popp and Harrison, 1969; Wolfe et al., 1969; Finegan and Harrison, 1970; Kostis and Moghadam, 1970; Nicholls et al., 1971; Nasser et al., 1972; Bass and Sharratt, 1973; Martinez et al., 1974; Sung et al., 1975; Zajtchuk et al., 1975; Petsas et al., 1976). 
In the case reported here echocardiography was helpful in excluding mitral stenosis but cardiac catheterisation was considered necessary to show conclusively that the abnormal echoes posterior to the anterior leaflet of the mitral valve and the aortic root (Fig. 1A and 1B) were the result of a left atrial tumour. These abnormal echoes were then interpreted as coming from the posterior surface of the myxoma itself as it moved in and out of the mitral valve orifice. Sung et al. (1975) showed the value of sweeping the ultrasonic beam along the longitudinal axis of the heart while continuously recording the echocardiogram in cases of left atrial myxoma. This technique was also useful in our case, which emphasises the value of its routine use in M-scan echocardiography.

The diagnosis of aortic regurgitation in the case described here was not made clinically. The fine fluttering observed in the mitral valve echogram (Fig. 1B) was subsequently interpreted as being the result of the aortic regurgitation (Winsberg et al., 1970) though on its own this would be insufficient to make the diagnosis. Aortic regurgitation was definitely present, however, as shown by root aortography (Fig. 2A and 2B), which was performed during a routine left and right heart catheterisation. This was undertaken because of the unusual echocardiographic presentation of this left atrial myxoma which to our knowledge had not previously been reported. Without the pulmonary angiogram and follow through, a diagnosis of mitral stenosis with trivial mitral regurgitation and moderate aortic regurgitation, all of rheumatic aetiology, might have been made. Because of the echocardiographic findings, however, pulmonary angiography was performed in this case to show the left atrial myxoma. The very important contribution of echocardiography in this case stresses the need for this noninvasive investigation to precede cardiac catheterisation and angiocardiography in all cases thought to have valvular heart disease.

In his review of left atrial myxoma, Goodwin (1963) mentioned a patient with coexistent aortic regurgitation but no details were given. The well-documented report of a case with left atrial myxoma and aortic regurgitation by Rose et al. (1974) is the only other record of this combination of lesions that we have found. These authors suggested that the aortic regurgitation in their case was caused by direct trauma to the aortic valve cusps. The tumour they described was a large lobulated mass $8 \mathrm{~cm}$ long which was demonstrated to fall through the mitral valve orifice in diastole to a point just below the aortic valve. In our patient the tumour was smaller and only a small part of it was seen to fall into the mitral valve orifice in diastole (Fig. 2C and 2D). The pedicle of the myxoma, however, was attached to that part of the septum adjacent to the aortic root, so that as the tumour fell through the mitral valve orifice it deformed the aortic root (Fig. 2A and 2B). We believe that it was this mechanical deformation of the aortic root by the tumour and its pedicle that caused the aortic regurgitation in our case, rather than direct trauma to the aortic valve cusps.

\section{References}

Bass, N. M., and Sharratt, G. P. (1973). Left atrial myxoma diagnosed by echocardiography with observations on tumour movement. British Heart fournal, 35, 1332-1335.

Effert, S., and Domanig, E. (1959). The diagnosis of intraatrial tumours and thrombi by the ultrasonic echo method. German Medical Monthly, 4, 1-3.

Finegan, R. E., and Harrison, D. C. (1970). Diagnosis of left atrial myxoma by echocardiography. New England Fournal of Medicine, 282, 1022-1023.

Goodwin, J. F. (1963). Diagnosis of left atrial myxoma. Lancet, 1, 464-468.

Kostis, J. B., and Moghadam, A. N. (1970). Echocardiographic diagnosis of left atrial myxoma. Chest, 58, 550-552.

Martinez, E. C., Giles, T. D., and Burch, G. E. (1974). Echocardiographic diagnosis of left atrial myxoma. American Fournal of Cardiology, 33, 281-285.

Nasser, W. K., Davis, R. H., Dillon, J. C., Tavel, M. E., Helmen, C. H., Feigenbaum, H., and Fisch, C. (1972). Atrial myxoma II. Phonocardiographic, echocardiographic hemodynamic and angiographic features in nine cases. American Heart fournal, 83, 810-824.

Nicholls, A. J. S., Goodman, M. A., and Pridie, R. B. (1971). Left atrial myxoma in a child diagnosed by ultrasound. Postgraduate Medical fournal, 47, 804-807.

Petsas, A. A., Gottlieb, S., Kingsley, B., Segal, B. L., and Myerburg, R. J. (1976). Echocardiographic diagnosis of left atrial myxoma. Usefulness of suprasternal approach. British Heart fournal, 38, 627-632.

Popp, R. L., and Harrison, D. C. (1969). Ultrasound for the diagnosis of atrial tumour. Annals of Internal Medicine, 71, 785-787.

Rose, M. R., Fox, A. C., Glassman, E., and Reed, G. E. (1974). Left atrial myxoma and aortic regurgitation. fournal of Thoracic and Cardiovascular Surgery, 68, 797801.

Schattenberg, T. T. (1968). Echocardiographic diagnosis of left atrial myxoma. Mayo Clinic Proceedings, 43, 620-627.

Sung, R. J., Ghahramani, A. R., Mallon, S. M., Richter, S. E., Sommer, L. S., Gottlieb, S., and Myerburg, R. J. (1975). Hemodynamic features of prolapsing and nonprolapsing left atrial myxoma. Circulation, 51, 342-349.

Winsberg, F., Gabor, G. E., Hernberg, J. G., and Weiss, B. (1970). Fluttering of the mitral valve in aortic insufficiency. Circulation, 41, 225-230.

Wolfe, S. B., Popp, R. L., and Feigenbaum, H. (1969). Diagnosis of atrial tumours by ultrasound. Circulation, 39, 615-622.

Zajtchuk, R., Fitterer, J. D., Strevey, T. E., and Nelson, W. P. (1975). Bilateral atrial myxomas. Pre-operative diagnosis and successful removal. Fournal of Thoracic and Cardiovascular Surgery, 69, 291-294.

Requests for reprints to Dr P. D. V. Bourdillon, National Heart Hospital, Westmoreland Street, London W1M 8BA. 